\title{
Examination News:
}

Result of FCPS Part-I, FCPS Part-II and MCPS Examinations held in July, 2008 are given below:

3464 candidates appeared in FCPS Part-I Examination held in July, 2008, of which 638 candidates came out successful, Subject- wise results are as follows:

\section{FCPS Part-I Examination:}

\begin{tabular}{llccc}
\hline SL No. & Name of the Speciality & No. of Candidates Appered & No. of Candidates Passed & \% of Pass \\
\hline 1. & Anaesthesiology & 47 & 5 & 10.64 \\
2. & Biochemistry & 2 & 0 & 0.00 \\
3. & Dentistry & 111 & 25 & 22.52 \\
4. & Dermatology \& Venereology & 90 & 28 & 31.11 \\
5. & Family Medicine & 5 & 0 & 0.00 \\
6. & Haematology & 26 & 3 & 11.54 \\
7. & Histopathology & 7 & 3 & 42.86 \\
8. & Medicine & 1083 & 162 & 14.96 \\
9. & Microbiology & 11 & 0 & 0.00 \\
10. & Obst. \& Gynae & 848 & 231 & 27.24 \\
11. & Ophthalmology & 90 & 7 & 7.78 \\
12. & Otolaryngology & 81 & 4 & 4.94 \\
13. & Paediatrics & 358 & 50 & 13.97 \\
14. & Physical Medicine \& Rehabilitation & 23 & 12 & 52.17 \\
15. & Psychiatry & 7 & 2 & 28.57 \\
16. & Radiology \& Imaging & 53 & 1 & 1.89 \\
17. & Radiotherapy & 18 & 8 & 44.44 \\
18. & Surgery & 600 & 97 & 16.17 \\
19. & Transfusion Medicine & 4 & 0 & 0.00 \\
\hline & Grand Total & 3464 & 638 & 18.42 \\
\hline
\end{tabular}

873 candidates appeared in FCPS Part-II Examination in Different subjects, List of candidates who satisfied the board of examiners is as follows:

\begin{tabular}{llll}
\hline Roll No. & Name of candidate & From where Graduated & Speciality \\
\hline $073-7003$ & Dr. Mohammad Raihan Uddin & MAG Osmani Medical College, Sylhet & Anaesthesiology \\
$073-7004$ & Dr. Satyajit Dhar & MAG Osmani Medical College, Sylhet & Anaesthesiology \\
$073-7008$ & Dr. Md. Anisur Rahman & Rajshahi Medical College, Rajshahi & Anaesthesiology \\
$073-7013$ & Dr. Md. Aminur Rahman & Rajshahi Medical College, Rajshahi & Anaesthesiology \\
$073-7014$ & Dr. Giashuddin Ahmed & Sher-e-Bangla Medical College, Barisal & Anaesthesiology \\
$073-7017$ & Dr. Rubaiya Ali & Chittagong Medical College, Chittagong & Dermatology \& Venereology \\
$073-7018$ & Dr. Ayesha Hassan & Dhaka Medical College, Dhaka & Dermatology \& Venereology \\
$073-7026$ & Dr. Rokeya Begum & Sher-e-Bangla Medical College, Barisal & Dermatology \& Venereology \\
$073-7030$ & Dr. Md. Motiul Hossain & Dhaka Medical College, Dhaka & Dermatology \& Venereology \\
$073-7037$ & Dr. A.K.M. Nurun Nobi & Rangpur Medical College, Rangpur & Dermatology \& Venereology \\
\hline
\end{tabular}




\begin{tabular}{|c|c|c|c|}
\hline Roll No. & Name of candidate & From where Graduated & Speciality \\
\hline 073-7041 & Dr. Tasneem Ara & & Haematology \\
\hline 073-7042 & Dr. Syed Sabbir Mahmood & Sher-e-Bangla Medical College, Barisal & Haematology \\
\hline 073-7046 & Dr. A.K.M. Kamruzzaman & Mymensing Medical College, Mymensing & Haematology \\
\hline 073-7055 & Dr. A B M Saiful Alam & Rajshahi Medical College, Rajshahi & Medicine \\
\hline 073-7058 & Dr. Mohammad Hasan Tarik & Rajshahi Medical College, Rajshahi & Medicine \\
\hline 073-7059 & Dr. Gobinda Chandra Roy & Mymensing Medical College, Mymensing & Medicine \\
\hline 073-7071 & Dr. F.M. Atiqur Rahman & Dhaka Medical College, Dhaka & Medicine \\
\hline 073-7075 & Dr. Rajib Barua & Dhaka Medical College, Dhaka & Medicine \\
\hline 073-7083 & Dr. Md. Azizul Hasan Khandaker & Mymensing Medical College, Mymensing & Medicine \\
\hline 073-7085 & Dr. Mostak Uddin Ahmed & Dhaka Medical College, Dhaka & Medicine \\
\hline 073-7097 & Dr. Utpal Kumar Chanda & Rangpur Medical College, Rangpur & Medicine \\
\hline 073-7103 & Dr. Syed Rahat Hassan & Sir Salimullah Medical College, Dhaka & Medicine \\
\hline 073-7108 & Dr. Md. Ariful Islam & Dhaka Medical College, Dhaka & Medicine \\
\hline 073-7109 & Dr. Md. Masudul Hassan & Rajshahi Medical College, Rajshahi & Medicine \\
\hline 073-7110 & Dr. Syed Ghulam Mogni Mowla & Chittagong Medical College, Chittagong & Medicine \\
\hline 073-7112 & Dr. Abu Shahin Mohammed Mahbubur Ra hman & Chittagong Medical College, Chittagong & Medicine \\
\hline $073-7115$ & Dr. Saleh Ahmed & Mymensing Medical College, Mymensing & Medicine \\
\hline $073-7153$ & Dr. Ahmed Shafiqul Hossain & Mymensing Medical College, Mymensing & Medicine \\
\hline $073-7165$ & Dr. Md. Rezaul Islam & Dhaka Medical College, Dhaka & Medicine \\
\hline 073-7169 & Dr. Manoranjan Roy & Sir Salimullah Medical College, Dhaka & Medicine \\
\hline $073-7170$ & Dr. Md. Quadrat-e-Elahi & Sir Salimullah Medical College, Dhaka & Medicine \\
\hline 073-7210 & Dr. Arifur Rahman & Dhaka Medical College, Dhaka & Medicine \\
\hline $073-7211$ & Dr. Dipankar Roy & Dhaka Medical College, Dhaka & Medicine \\
\hline $073-7225$ & Dr. Ahmad Hossen Morshed & Chittagong Medical College, Chittagong & Medicine \\
\hline $073-7228$ & Dr. Farhana Moslehuddin & Dhaka Medical College, Dhaka & Medicine \\
\hline $073-7233$ & Dr. Mohammad Kawser Uddin & Comilla Medical College, Comilla & Medicine \\
\hline $073-7238$ & Dr. Azizun Nessa & Dhaka Medical College, Dhaka & Medicine \\
\hline 073-7239 & Dr. Md Enayet Hussain & Dhaka Medical College, Dhaka & Medicine \\
\hline 073-7269 & Dr. Mohammad Abul Hasnat & MAG Osmani Medical College, Sylhet & Medicine \\
\hline $073-7278$ & Dr. Mohammad Ibrahim Khalil & Mymensing Medical College, Mymensing & Medicine \\
\hline 073-7279 & Dr. Masum Ahmed & Sher-e-Bangla Medical College, Barisal & Medicine \\
\hline $073-7287$ & Dr. Sharmin Jahan & Dhaka Medical College, Dhaka & Medicine \\
\hline 073-7298 & Dr. Mst. Marfia Khatun & Dhaka Medical College, Dhaka & Obst and Gynae \\
\hline $073-7323$ & Dr. Iffat Zaman & Sir Salimullah Medical College, Dhaka & Obst and Gynae \\
\hline 073-7366 & Dr. Junnu Rayen Janna & Rajshahi Medical College, Rajshahi & Obst and Gynae \\
\hline 073-7397 & Dr. Taslim Nila & Dhaka Medical College, Dhaka & Obst and Gynae \\
\hline 073-7411 & Dr. Fahmida Rashid & Jahurul Islam Medical College, Bajitpur & Obst and Gynae \\
\hline 073-7412 & Dr. Mst. Meher Afroz & Sir Salimullah Medical College, Dhaka & Obst and Gynae \\
\hline $073-7420$ & Dr. Nargis Nahar & Sir Salimullah Medical College, Dhaka & Obst and Gynae \\
\hline 073-7440 & Dr. Shamim Ara Hoque & Institte of Applied Health Science,Under USTC, Chittagong & Obst and Gynae \\
\hline $073-7446$ & Dr. Nibash Chandra Paul & MAG Osmani Medical College, Sylhet & Obst and Gynae \\
\hline $073-7452$ & Dr. Ayesha Siddika & Rajshahi Medical College, Rajshahi & Obst and Gynae \\
\hline $073-7453$ & Dr. Shaida Akhter & Rangpur Medical College, Rangpur & Obst and Gynae \\
\hline $073-7460$ & Dr. Zeba Ahmed & MAG Osmani Medical College, Sylhet & Obst and Gynae \\
\hline $073-7463$ & Dr. Ferdousi Begum & Rajshahi Medical College, Rajshahi & Obst and Gynae \\
\hline
\end{tabular}




\begin{tabular}{|c|c|c|c|}
\hline Roll No. & Name of candidate & From where Graduated & Speciality \\
\hline $073-7474$ & Dr. Mousumi Kader & Khulna Medical College, Khulna & Obst and Gynae \\
\hline $073-7476$ & Dr. Rokeya Ferdousey & Rajshahi Medical College, Rajshahi & Obst and Gynae \\
\hline 073-7490 & Dr. Nasreen Afreen & Sher-e-Bangla Medical College, Barisal & Obst and Gynae \\
\hline 073-7491 & Dr. Jesmin Jerin & Sir Salimullah Medical College, Dhaka & Obst and Gynae \\
\hline 073-7495 & Dr. Begum Shamsun Nahar Kana & Mymensing Medical College, Mymensing & Obst and Gynae \\
\hline 073-7498 & Dr. Farhana Begum & Rangpur Medical College, Rangpur & Obst and Gynae \\
\hline 073-7502 & Dr. Shamsun Nahar & Sher-e-Bangla Medical College, Barisal & Obst and Gynae \\
\hline 073-7507 & Dr. Maksuda Akhter & Sir Salimullah Medical College, Dhaka & Obst and Gynae \\
\hline 073-7511 & Dr. Fahmida Haque & Sir Salimullah Medical College, Dhaka & Obst and Gynae \\
\hline $073-7520$ & Dr. Kishuar Parveen & MAG Osmani Medical College, Sylhet & Obst and Gynae \\
\hline $073-7526$ & Dr. Mst. Farhana Tarannum Khan & Rajshahi Medical College, Rajshahi & Obst and Gynae \\
\hline 073-7529 & Dr. Md. Abdul Halim & Rangpur Medical College, Rangpur & Opthalmology \\
\hline $073-7530$ & Dr. Zahidul Ahsan & Sher-e-Bangla Medical College, Barisal & Opthalmology \\
\hline 073-7541 & Dr. Md. Naimul Haque & Rajshahi Medical College, Rajshahi & Opthalmology \\
\hline $073-7548$ & Dr. Md. Shahidul Islam & Rajshahi Medical College, Rajshahi & Opthalmology \\
\hline 073-7550 & Dr. Md. Anisuzzaman & Dhaka Medical College, Dhaka & Opthalmology \\
\hline $073-7553$ & Dr. Md. Nurul Islam & Rajshahi Medical College, Rajshahi & Opthalmology \\
\hline $073-7555$ & Dr. Bilkis Begum & Dhaka Medical College, Dhaka & Opthalmology \\
\hline 073-7566 & Dr. Fatema Akhter & Dhaka Dental College, Dhaka & Orthodontics and Dentofacial Orthopaedics \\
\hline 073-7569 & Dr. Abdul- Qayum-Chowdhury & Chittagong Medical College, Chittagong & Otolaryngology \\
\hline 073-7584 & Dr. S.M. Mesbah Uddin Ahmed & Sher-e-Bangla Medical College, Barisal & Otolaryngology \\
\hline $073-7586$ & Dr. Mohammad Ashequr Rahman Bhuiyan & Dhaka Medical College, Dhaka & Otolaryngology \\
\hline $073-7597$ & Dr. Mohammad Anwar Hossain & Dhaka Medical College, Dhaka & Otolaryngology \\
\hline 073-7599 & Dr. Md. Iftekhar-UL-Haque Khan & Dhaka Medical College, Dhaka & Paediatrics \\
\hline 073-7601 & Dr. Nilufa Parvin & Dhaka Medical College, Dhaka & Paediatrics \\
\hline 073-7605 & Dr. Naima Sharmin & Dhaka Medical College, Dhaka & Paediatrics \\
\hline 073-7610 & Dr. Nasim Jahan & MAG Osmani Medical College, Sylhet & Paediatrics \\
\hline 073-7613 & Dr. Farzana Hamid & Dhaka Medical College, Dhaka & Paediatrics \\
\hline 073-7614 & Dr. Farhana Jalil & Sir Salimullah Medical College, Dhaka & Paediatrics \\
\hline $073-7615$ & Dr. Khan Lamia Nahid & Dhaka Medical College, Dhaka & Paediatrics \\
\hline 073-7618 & Dr. Asma Ferdousi & Chittagong Medical College, Chittagong & Paediatrics \\
\hline 073-7622 & Dr. Shamima Naznin & Sir Salimullah Medical College, Dhaka & Paediatrics \\
\hline $073-7624$ & Dr. Fatema Begum & MAG Osmani Medical College, Sylhet & Paediatrics \\
\hline 073-7649 & Dr. Shirmin Ara Ferdousi & Sir Salimullah Medical College, Dhaka & Paediatrics \\
\hline $073-7656$ & Dr. Md. Atiar Rahman & Dhaka Medical College, Dhaka & Paediatrics \\
\hline 073-7657 & Dr. Muhammad Solaiman Mollah & Sir Salimullah Medical College, Dhaka & Paediatrics \\
\hline 073-7659 & Dr. Md. Abu Sufian & MAG Osmani Medical College, Sylhet & Paediatrics \\
\hline 073-7674 & Dr. Mustafa Mahbub & Sher-e-Bangla Medical College, Barisal & Paediatrics \\
\hline 073-7684 & Dr. Susanta Kumer Ghosh & Sir Salimullah Medical College, Dhaka & Paediatrics \\
\hline 073-7691 & Dr. Utpol Chowdhury & Mymensing Medical College, Mymensing & Paediatrics \\
\hline 073-7693 & Dr. Bidhan Chandra Biswas & Sher-e-Bangla Medical College, Barisal & Paediatrics \\
\hline 073-7700 & Dr. Bikash Chandra Nag & Sher-e-Bangla Medical College, Barisal & Paediatrics \\
\hline 073-7708 & Dr. Tahmina Jesmin & MAG Osmani Medical College, Sylhet & Paediatrics \\
\hline 073-7709 & Dr. Ahmed Sayeed & Sir Salimullah Medical College, Dhaka & Paediatrics \\
\hline 073-7710 & Dr. Manzur Ahmed & MAG Osmani Medical College, Sylhet & Paediatrics \\
\hline
\end{tabular}




\begin{tabular}{|c|c|c|c|}
\hline Roll No. & Name of candidate & From where Graduated & Speciality \\
\hline $073-7712$ & Dr. Md. Ashraful Islam & Rangpur Medical College, Rangpur & Paediatrics \\
\hline $073-7715$ & Dr. Md. Delwar Hossain & Sir Salimullah Medical College, Dhaka & Paediatrics \\
\hline 073-7717 & Dr. Taslima Hoq Moonmoon & Dhaka Medical College, Dhaka & Physical Medicine \& Rehabilitation \\
\hline 073-7721 & Dr. Md. Amzad Hossain & Dhaka Dental College, Dhaka & Prosthodontics \\
\hline $073-7725$ & Dr. Robindra Nath Sarker & Dhaka Medical College, Dhaka & Radiology \& Imaging \\
\hline $073-7726$ & Dr. Osmanur Rashid & Sir Salimullah Medical College, Dhaka & Radiology \& Imaging \\
\hline $073-7727$ & Dr. Zebun Nahar & MAG Osmani Medical College, Sylhet & Radiology \& Imaging \\
\hline $073-7737$ & Dr. Nasir Uddin Mahmud & Comilla Medical College, Comilla & Surgery \\
\hline $073-7743$ & Dr. Farid Ahmed & Mymensing Medical College, Mymensing & Surgery \\
\hline $073-7750$ & Dr. Md. Waliur Rahman & Rajshahi Medical College, Rajshahi & Surgery \\
\hline 073-7756 & Dr. Gobinda Chandra Saha & Sir Salimullah Medical College, Dhaka & Surgery \\
\hline $073-7763$ & Dr. Md. Maniul Hasan & Dhaka Medical College, Dhaka & Surgery \\
\hline 073-7764 & Dr. Rajkumar Roy & Rangpur Medical College, Rangpur & Surgery \\
\hline 073-7777 & Dr. Md. Siddiqur Rahman & Sher-e-Bangla Medical College, Barisal & Surgery \\
\hline 073-7806 & Dr. Md. Atiar Rahman & Dhaka Medical College, Dhaka & Surgery \\
\hline $073-7820$ & Dr. Ferdous Alam & Dhaka Medical College, Dhaka & Surgery \\
\hline $073-7826$ & Dr. Khandkar Ali Kawsar & Dhaka Medical College, Dhaka & Surgery \\
\hline 073-7844 & Dr. Md. Monoar Hossain & Rajshahi Medical College, Rajshahi & Surgery \\
\hline 073-7846 & Dr. Mohammad Monir Hossain Bhuiyan & Khulna Medical Collge, Khulna & Surgery \\
\hline 073-7849 & Dr. Md. Abdul Mazid & Chittagong Medical College, Chittagong & Surgery \\
\hline 0737858 & Dr. Ehsanur Reza & Dhaka Medical College, Dhaka & Surgery \\
\hline $073-7862$ & Dr. Muhammad Humayun Kabir & Sir Salimullah Medical College, Dhaka & Surgery \\
\hline $073-7863$ & Dr. Md. Monowarul Islam & Sher-e-Bangla Medical College, Barisal & Surgery \\
\hline $073-7867$ & Dr. Md. Ashif Chowdhury & Rajshahi Medical College, Rajshahi & Surgery \\
\hline 073-7871 & Dr. Shagorika Sharmeen & Chittagong Medical College, Chittagong & Surgery \\
\hline $012-8501$ & Dr. A F M Shamsul Haque & Mymensing Medical College, Mymensing & Cardiology \\
\hline $012-8503$ & Dr. Mohammed Shadrul Alam & Chittagong Medical College, Chittagong & Paediatric Surgery \\
\hline $012-8505$ & Dr. S M Shameem Waheed & Sher-e-Bangla Medical College, Barisal & Urology \\
\hline
\end{tabular}

67 candidates appeared in Priliminary FCPS- II Examination in different subjects. List of candidates who satisfied the board of examiners is as follows:

\begin{tabular}{llll}
\hline Roll No. & Name of candidate & From where Graduated & Speciality \\
\hline $007-8001$ & Dr. Shaila Nabi & Sir Salimullah Medical College, Dhaka & Preli-Medicine \\
$007-8002$ & Dr. Tanjima Parvin & Dhaka Medical College, Dhaka & Preli-Medicine \\
$007-8007$ & Dr. Sabina Hashem & Chittagong Medical College, Chittagong & Preli-Medicine \\
$007-8010$ & Dr. Jalal Mohsin Uddin & Jahurul Islam Medical College, Bajitpur & Preli-Medicine \\
$007-8011$ & Dr. S.M. Lutfor Rahman & Sher-e-Bangla Medical College, Barisal & Preli-Medicine \\
$007-8017$ & Dr. Sharafat Nurul Islam & Dhaka Medical College, Dhaka & Preli-Medicine \\
$007-8020$ & Dr. Md. Arif Akbar Saibal & Rajshahi Medical College, Rajshahi & Preli-Medicine \\
$007-8046$ & Dr. Mohammad Serajus Saleheen & Mymensing Medical College, Mymensing & Preli-Surgery \\
$007-8049$ & Dr. Md. Meraj Uddin Mollah & Dhaka Medical College, Dhaka & Preli-Surgery \\
$007-8057$ & Dr. Farzana Bilquis Ibrahim & Bangladesh Medical College, Dhaka & Preli-Surgery \\
$007-8061$ & Dr. Mirza Mohammad Tyeabul Islam & Faridpur Medical College, Faridpur & Preli-Surgery \\
\hline
\end{tabular}


290 candidates appeared in MCPS Examination in different subjects. List of candidates who satisfied the board of examiners is as follows:

\begin{tabular}{|c|c|c|c|}
\hline Roll No. & Name of candidate & From where Graduated & Speciality \\
\hline 073-9001 & Dr. Siba Pada Roy & Rangpur Medical College, Rangpur & Anaesthesiology \\
\hline 073-9005 & Dr. ATM. Rashidun Nabi & Rangpur Medical College, Rangpur & Anaesthesiology \\
\hline 073-9006 & Dr. Sharif Md. Abu Sayeed & Rajshahi Medical College, Rajshahi & Anaesthesiology \\
\hline 073-9007 & Dr. Apple Mahmud Anwar & Jahurul Islam Medical College, Bajitpur & Anaesthesiology \\
\hline 073-9008 & Dr. Kazi Reajul Islam & Rangpur Medical College, Rangpur & Anaesthesiology \\
\hline 073-9010 & Dr. Abdullah Masum & Khulna Medical Collge, Khulna & Anaesthesiology \\
\hline 073-9012 & Dr. Mohammad Shaheen Miah & Institute of Applied Health Science, Under USTC, Chittagong & Anaesthesiology \\
\hline 073-9017 & Dr. Farida Arjuman & Chittagong Medical College, Chittagong & Clinical Pathology \\
\hline 073-9020 & Dr. Md. Abdur Rauf & Dhaka Dental College, Dhaka & Dental Surgery \\
\hline 073-9021 & Dr. Zahid-Ul-Alam Shaikh & Dhaka Dental College, Dhaka & Dental Surgery \\
\hline $073-9025$ & Dr. Nazmus Sadat & Dhaka Dental College, Dhaka & Dental Surgery \\
\hline 073-9026 & Dr. SK. Nazrul Islam & Dhaka Dental College, Dhaka & Dental Surgery \\
\hline 073-9028 & Dr. Muhammed Ashraful Alam & Chittagong Medical College, Chittagong & Dermatology and Venerelogy \\
\hline 073-9029 & Dr. Mohammad Aminul Islam & MAG Osmani Medical College, Sylhet & Dermatology and Venerelogy \\
\hline 073-9033 & Dr. Lubna Khondker & Rangpur Medical College, Rangpur & Dermatology and Venerelogy \\
\hline 073-9045 & Dr. Chandan Kumar Saha & Dhaka Medical College, Dhaka & Medicine \\
\hline 073-9052 & Dr. Shakib-UZ-Zaman Arefin & Sir Salimullah Medical College, Dhaka & Medicine \\
\hline 073-9053 & Dr. Md. Wahedul Haque & Rajshahi Medical College, Rajshahi & Medicine \\
\hline 073-9065 & Dr. Mohammad Kamal Uddin & Dhaka Medical College, Dhaka & Medicine \\
\hline 073-9079 & Dr. Mohammed Arshad-Ul-Azim & Chittagong Medical College, Chittagong & Medicine \\
\hline 073-9084 & Dr. Abul Kalam Mohammed Shoab & Sir Salimullah Medical College, Dhaka & Medicine \\
\hline 073-9085 & Dr. A.H.M. Aktaruzzaman & Sir Salimullah Medical College, Dhaka & Medicine \\
\hline 073-9103 & Dr. Mohd. Rumi Alam & MAG Osmani Medical College, Sylhet & Medicine \\
\hline 073-9106 & Dr. Md. Rezaul Karim & Rajshahi Medical College, Rajshahi & Medicine \\
\hline 073-9112 & Dr. A K M Humayon Kabir & Dhaka Medical College, Dhaka & Medicine \\
\hline 073-9121 & Dr. Rumana Arman & Medical College for Women and Hospital, Dhaka & Obst and Gynae \\
\hline 073-9122 & Dr. Syeeda Banu & Rangpur Medical College, Rangpur & Obst and Gynae \\
\hline 073-9126 & Dr. Sakila Khatun & Mymensing Medical College, Mymensing & Obst and Gynae \\
\hline 073-9129 & Dr. Shamsun Nahar Tania & MAG Osmani Medical College, Sylhet & Obst and Gynae \\
\hline 073-9130 & Dr. Parveen Akter & Chittagong Medical College, Chittagong & Obst and Gynae \\
\hline 073-9131 & Dr. Apurba Kumar Biswas & Rajshahi Medical College, Rajshahi & Obst and Gynae \\
\hline 073-9132 & Dr. Shahida Akter & Bangladesh Medical College, Dhaka & Obst and Gynae \\
\hline 073-9136 & Dr. Somaya Sidratul Montaha & Sir Salimullah Medical College, Dhaka & Obst and Gynae \\
\hline 073-9137 & Dr. Qumrun Nassa Ahmed & Bangladesh Medical College, Dhaka & Obst and Gynae \\
\hline 073-9143 & Dr. Farhana Jannat & MAG Osmani Medical College, Sylhet & Obst and Gynae \\
\hline 073-9146 & Dr. Farhat Banu & Z.H Shikder Women's Medical College, Dhaka & Obst and Gynae \\
\hline $073-9147$ & Dr. Rashida Akther & Chittagong Medical College, Chittagong & Obst and Gynae \\
\hline 073-9148 & Dr. Mafruha Khanam & Sir Salimullah Medical College, Dhaka & Obst and Gynae \\
\hline
\end{tabular}




\begin{tabular}{llll}
\hline Roll No. & Name of candidate & From where Graduated & Speciality \\
\hline $073-9157$ & Dr. Naimah Masood & Bangladesh Medical College, Dhaka & Obst and Gynae \\
$073-9165$ & Dr. Farzana Amir & Dhaka Medical College, Dhaka & Obst and Gynae \\
$073-9175$ & Dr. Mst. Amena Rahman & Comilla Medical College, Comilla & Obst and Gynae \\
$073-9176$ & Dr. Bilquis Fatema & Bangladesh Medical College, Dhaka & Obst and Gynae \\
$073-9184$ & Dr. Zakia Sultana & Mymensing Medical College, Mymensing & Obst and Gynae \\
$073-9189$ & Dr. Armana Islam & Bangladesh Medical College, Dhaka & Obst and Gynae \\
$073-9217$ & Dr. Rowshan Ara Begum & Dhaka Medical College, Dhaka & Obst and Gynae \\
$073-9223$ & Dr. Md. Nurun Nabi & Rajshahi Medical College, Rajshahi & Opthalmology \\
$073-9227$ & Dr. A.K.M. Mohsin Alam & & Opthalmology \\
$073-9228$ & Dr. Rinku Paul & Rangpur Medical College, Rangpur & Opthalmology \\
$073-9247$ & Dr. Zahir Sadique & Khulna Medical College, Khulna & Paediatrics \\
$073-9249$ & Dr. Mamun Reza Khan & Sher-e-Bangla Medical College, Barisal & Paediatrics \\
$073-9253$ & Dr. Niaz Mohammad Khan & Sir Salimullah Medical College, Dhaka & Psychiatry \\
$073-9254$ & Dr. Mubin Uddin Akhanji & Dhaka Medical College, Dhaka & Psychiatry \\
$073-9256$ & Dr. Md. Quamrul Islam & Dhaka Medical College, Dhaka & Radiology \& Imaging \\
$073-9257$ & Dr. Mohammed Mominul Hoque & Sher-e-Bangla Medical College, Barisal & Radiology \& Imaging \\
$073-9269$ & Dr. Md. Abdul Mannan & Sir Salimullah Medical College, Dhaka & Surgery \\
$073-9273$ & Dr. Abul Ata Md. Mozassem & Chittagong Medical College, Chittagong & Surgery \\
$073-9278$ & Dr. Quazi Habibullah & Dhaka Medical College, Dhaka & Surgery \\
\hline & & & \\
0
\end{tabular}


Continuing Professionals Development Lectures during April to June, 2008

\begin{tabular}{|c|c|c|c|c|}
\hline Date & Time & Topic & Speaker & Chairperson / Moderator \\
\hline $\begin{array}{l}\text { 06-05-2008 } \\
\text { Tuesday }\end{array}$ & $\begin{array}{l}11-00 \mathrm{am} \text { to } \\
11-50 \mathrm{am} \\
11-50 \mathrm{am} \text { to } \\
12-10 \mathrm{pm} \\
12-10 \mathrm{pm} \text { to } \\
1-00 \mathrm{pm}\end{array}$ & $\begin{array}{l}\text { TEA } \\
\text { "Pregnency with } \\
\text { Diabetes Mellitus" }\end{array}$ & $\begin{array}{l}\text { Prof. Md. Saleh Uddin } \\
\text { Chairman } \\
\text { Department of } \\
\text { Opthalmology } \\
\text { BSMMU, Dhaka. }\end{array}$ & $\begin{array}{l}\text { Prof. Md. Humayun Kabir } \\
\text { (Chairperson) } \\
\text { Prof. of Opthalmology } \\
\text { Prof. Md. Abdul Hadi Faquir } \\
\text { (Chairperson) } \\
\text { Assoc.Prof. of Opthalmology } \\
\text { Dr. Md. Musharaf Hossain } \\
\text { (Moderator) } \\
\text { Asstt. Prof. of Ophthalmology } \\
\text { Professor Sadiqa Tahera Khanam } \\
\text { (Chairperson) } \\
\text { Prof. of Obst. \& Gynae( Retd.) } \\
\text { Professor Mahmuda Khatun } \\
\text { (Chairperson) } \\
\text { Professor of Obst. \& Gynae } \\
\text { Dr. Ashia Khatun( Moderator) } \\
\text { Senior Consultant of Obst. \& Gynae }\end{array}$ \\
\hline $\begin{array}{l}\text { 13-05-2008 } \\
\text { Tuesday }\end{array}$ & $\begin{array}{l}11-00 \mathrm{am} \text { to } \\
11-50 \mathrm{pm} \\
11-50 \mathrm{am} \text { to } \\
12-10 \mathrm{pm} \\
12-10 \mathrm{pm} \text { to } \\
1-00 \mathrm{pm}\end{array}$ & $\begin{array}{l}\text { "Pregnancy with } \\
\text { Idiopathic } \\
\text { Thrombocytopenic } \\
\text { Purpura." } \\
\text { TEA } \\
\text { " Umbillical Cord } \\
\text { blood ( Stem cell) } \\
\text { Banking." }\end{array}$ & $\begin{array}{l}\text { Dr. Rabeya Akther } \\
\text { Senior Medical Officer } \\
\text { Bangladesh Bank } \\
\text { Medical Center } \\
\text { Motijheel, Dhaka. }\end{array}$ & $\begin{array}{l}\text { Professor Rowshan Ara Begum (Chair) } \\
\text { Professor of Obst. \& Gynae } \\
\text { Professor Sameena Chowdhury (Chair) } \\
\text { Professor \& Head, Dept. of Obst. \& Gynae } \\
\text { Dr. Tabassum Parveen (Moderator) } \\
\text { Assistant Professor of Obst. \& Gynae } \\
\text { Dr. Shahin Akhter Zahan Habib } \\
\text { (Co-Odinator) } \\
\text { Assistant Prof. of Obst. \& Gynae } \\
\text { Professor Firoza Begum (Chair) } \\
\text { Professor \& Head, Fetomaternal Medicine } \\
\text { Professor Saleha Begum Chowdhury (C) } \\
\text { Professor of Obst. \& Gynae } \\
\text { Dr. Rashida Khanom (Moderator) } \\
\text { Dr. Md. Atiar Rahman (Co-ordinator) } \\
\text { Assistant Professor of Surgery }\end{array}$ \\
\hline
\end{tabular}




\begin{tabular}{|c|c|c|c|c|}
\hline Date & Time & Topic & Speaker & Chairperson / Moderator \\
\hline $\begin{array}{l}\text { 27-05-2008 } \\
\text { Tuesday }\end{array}$ & $\begin{array}{l}11-00 \mathrm{am} \text { to } \\
11-50 \mathrm{am} \\
11-50 \mathrm{am} \text { to } \\
12-10 \mathrm{pm} \\
12-10 \mathrm{pm} \text { to } \\
1.00 \mathrm{pm}\end{array}$ & $\begin{array}{l}\text { "Doctor Patient } \\
\text { Relationship } \\
\text { "TEA" } \\
\text { Whether obesity } \\
\text { need to be } \\
\text { addressed as } \\
\text { pregnancy advice" }\end{array}$ & $\begin{array}{l} \\
\text { Dr. SK. Zinnat Ara Nasreen } \\
\text { Asso Prof \& Head Gyne } \\
\text { \& Obs } \\
\text { ZHSWMCH } \\
\text { Dhanmondi, Road No-10-A } \\
\text { House no-40, Flat no C3 } \\
\text { Lausel Spring, Dhaka-1209 }\end{array}$ & $\begin{array}{l}\text { Professor Syed Serajul Karim (Chair) } \\
\text { Professor of Surgery } \\
\text { Brig. Gen. (Retd.) Muhammad } \\
\text { Jahangir Hossain (Chair) } \\
\text { Professor \& Head of the Department } \\
\text { of Surgery } \\
\text { Dr. Md. Azharul Islam (Moderator) } \\
\text { Assistant Resident Surgeon } \\
\text { Dr. Md. Manir Hossain Khan (Co-ordin) } \\
\text { Assistant Prof. of Surgery } \\
\text { Dr. Nusrat Zaman (Chair) } \\
\text { Associate Professor of Obst. \& Gynae } \\
\text { Professor Khwaja Nazim Uddin (Chair) } \\
\text { Professor of Medicine } \\
\text { Dr. Tahmina Begum (Moderator) } \\
\text { Assistant Prof. of Obst. \& Gynae }\end{array}$ \\
\hline $\begin{array}{l}\text { 03-06-2008 } \\
\text { Tuesday }\end{array}$ & $\begin{array}{l}11-00 \mathrm{am} \text { to } \\
11-50 \mathrm{am}\end{array}$ & $\begin{array}{l}\text { "Study of Dapplev } \\
\text { Umbilical Artery } \\
\text { Velocimetny during } \\
\text { Third trimister of } \\
\text { Pregnancy. } \\
\text { TEA }\end{array}$ & $\begin{array}{l}\text { Dr. Nasreen Sultana } \\
\text { Registar } \\
\text { Obstetries \& } \\
\text { Gynaecology Departm } \\
\text { Dhaka National Medical } \\
\text { College } \\
\text { Dhaka. }\end{array}$ & $\begin{array}{l}\text { Professor Maliha Rashid (Chair) } \\
\text { Professor of Obst. \& Gynae } \\
\text { Dr. Ferdousi Islam (Chair) } \\
\text { Associate Professor of Obst. \& Gynae } \\
\text { Dr. Masuda Islam Khan (Moderator) } \\
\text { Registrar (Obst. \& Gynae), Unit-III } \\
\text { BIRDEM Hospital, Shabagh, Dhaka. } \\
\text { Dr. A.B.M. Bayezid Hossain } \\
\text { (Co-ordinator) } \\
\text { Assistant Prof. of Surgery }\end{array}$ \\
\hline
\end{tabular}




\begin{tabular}{|c|c|c|c|c|}
\hline Date & Time & Topic & Speaker & Chairperson / Moderator \\
\hline $\begin{array}{l}\text { 10-06-2008 } \\
\text { Tuesday }\end{array}$ & $\begin{array}{l}11-00 \mathrm{am} \text { to } \\
11-50 \mathrm{am}\end{array}$ & $\begin{array}{l}\text { "Treatment of } \\
\text { ruptured aneurysm, } \\
\text { Our experience" } \\
\text { TEA } \\
\text { "Screening for } \\
\text { Cervical cancer \& } \\
\text { the situation of } \\
\text { Bangladesh." }\end{array}$ & $\begin{array}{l}\text { Prof. Kanak Kanti Barua } \\
\text { Prof. of Neurosurgery } \\
\text { BSMMU, Shahbagh, } \\
\text { Dhaka. }\end{array}$ & $\begin{array}{l}\text { Professor Md. Wahiduzzaman (Chair) } \\
\text { Consultant, Neuro-Surgery } \\
\text { Professor Mohammad Afzal Hossain (Chair) } \\
\text { Chairman \& Professor of Neuro-Surgery } \\
\text { Dr. Zahed Ali (Moderator) } \\
\text { Dr. Md. Ayub Al Mamun (Co-ordinator) } \\
\text { Assistant Professor of Medicine } \\
\text { Professor Sultana Razia Begum (Chair) } \\
\text { Professor of Obst. \& Gynae } \\
\text { Professor Mst. Sabera Khatun (Chair) } \\
\text { Professor of Obst. \& Gynae } \\
\text { Dr. Meherun Nessa (Moderator) } \\
\text { Assistant Prof. of Obst \& Gynae } \\
\text { Dr. Mohammad Mohibur Rahman (Co-ord) } \\
\text { Assistant Prof. of Medicine }\end{array}$ \\
\hline $\begin{array}{l}\text { 17-06-2008 } \\
\text { Tuesday }\end{array}$ & $\begin{array}{l}11-00 \mathrm{am} \text { to } \\
11-50 \mathrm{am} \\
11-50 \mathrm{am} \text { to } \\
12-10 \mathrm{pm} \\
12-10 \mathrm{pm} \text { to } \\
1.00 \mathrm{pm}\end{array}$ & $\begin{array}{l}\text { Breast pain in } \\
\text { female: } \\
\text { Underdiagnosed or } \\
\text { overdiagnosed? } \\
\text { TEA } \\
\text { " Corneal ulcer } \\
\text { Diagnosis, } \\
\text { Management and } \\
\text { Prevention } \\
\text { Bangladesh } \\
\text { Perspective." }\end{array}$ & $\begin{array}{l}\text { Prof. Anisur Rahman } \\
\text { MBBS, MS, FCPS, FRCS } \\
\text { Consultant and Coordinator } \\
\text { Dept of Gen and Lap } \\
\text { Surgery } \\
\text { Apollo Hospitals, Dhaka. }\end{array}$ & $\begin{array}{l}\text { Dr. (Major General) A.K.M. Zafrullah Siddiq } \\
\text { (Chair) } \\
\text { Consultant Surgeon General (Chair } \\
\text { Professor Md. Shahid Hossain (Chair) } \\
\text { Professor \& Head, Dept. of Surgical Oncology } \\
\text { Dr. Md. Fazal Naser (Moderator) } \\
\text { Dr. Mohd. Zahid Hussain (Co-ordinator) } \\
\text { Associate Professor of Paediatrics } \\
\text { Professor A.S.M. Kamal Uddin (Chair) } \\
\text { Director cum Professor } \\
\text { MIA Institute of Ophthalmology } \\
\text { Dr. Col. Md. Anwar Hossain (Chair) } \\
\text { Classified Specialist in Ophthalmology } \\
\text { Dr. Iftekhar Md. Munir (Moderator) } \\
\text { Eye Specialist } \\
\text { Dr. Syed Abdul Wadud (Moderator) } \\
\text { Asstt. Professor of Ophthamology }\end{array}$ \\
\hline
\end{tabular}

